\title{
MIMO OFDM Implementation in Cognitive Scenario
}

\author{
Rahul Gedam $^{1}$, Dr. M. R. Meshram ${ }^{2}$, Dr. A. K. Shrivastava ${ }^{3}$ \\ PhD. Scholar, Department of Electronics, C. V. R. University Bilaspur (C.G) India \\ Professor, Department of ET\&T, GEC, Jagdalpur(C.G)India
}

Professor, Department of Physics, C.V. R. University Bilaspur (C.G) India

\begin{abstract}
Cognitive; meaning concerned with the act or process of knowing, perceiving etc. cognitive radio CR is a concept of creating a software defined Radio platform for utilizing the spectrum holes which are underutilized. By smartly using the spectrum the scarcity of spectrum problem and congestion of data traffic in a network can be minimized in a very sophisticated manner. Orthogonal frequency division multiplexing is a reliable and authentic transmission system for CR system which provides us with great amount of flexibility to fully utilized the spectrum in the active mode. Dynamically using the available spectrum resources is the highlight of the paper, when we think of an efficient communication module there are various parameters which has to be accounted for e.g. mutual interference, data rate, band width utilization, bit error rate, signal to noise ratio etc. This paper presents a MIMO-OFDM structure in cognitive radio scenario, performance of proposed system is evaluated on the basis of Bit error rate (BER) and signal to noise ratio (SNR).
\end{abstract}

Keywords: Cognitive radio, OFDM, OFDM-MIMO, Bit error rate, Signal to noise ratio

\section{Introduction}

OFDM system transmits high rate data over a number of low rate overlapping multi-carriers, which are multiplexed in frequency domain. To realize the overlapping design, we have to guarantee the mathematical orthogonality to reduce the crosstalk between multi-carriers. In the traditional nonoverlapping FDM (Frequency Division Multiplex) system, many carriers should be spaced apart to guarantee the orthogonality between adjacent carriers, and guard bands, which lower the spectrum efficiency, are required in addition. However, OFDM system can save the bandwidth by using overlapping orthogonal multi-carriers. Furthermore, OFDM system takes the robustness against frequency-selective fading by making fading effect on subcarriers almost frequency-flat. To mitigate ISI (InterSymbol Interference) by multipath channels, CP (Cyclic Prefix) is inserted before transmitting the signal over the $M_{T}$ Transmit antermas radio channel. The implementation complexity of $\mathrm{CP}$ OFDM to overcome multipath fading is lower than those of systems using equalizers.

MIMO-OFDM system, which means that OFDM system is combined with MIMO technologies, is very promising to support the high data rate transmission for the nextgeneration wireless communication systems by taking advantages of two powerful technologies.

Multiple-Input Multiple-Output (MIMO) system is a major topic of research over a few decades because of high spectral efficiency and reliability of performance in a given bandwidth offered by them. In such systems, the goal is to minimize the Bit Error Rate (BER) for a given Signal-toNoise Ratio (SNR) while maintaining reliability of communication and keeping in mind the complexity and cost of the system.

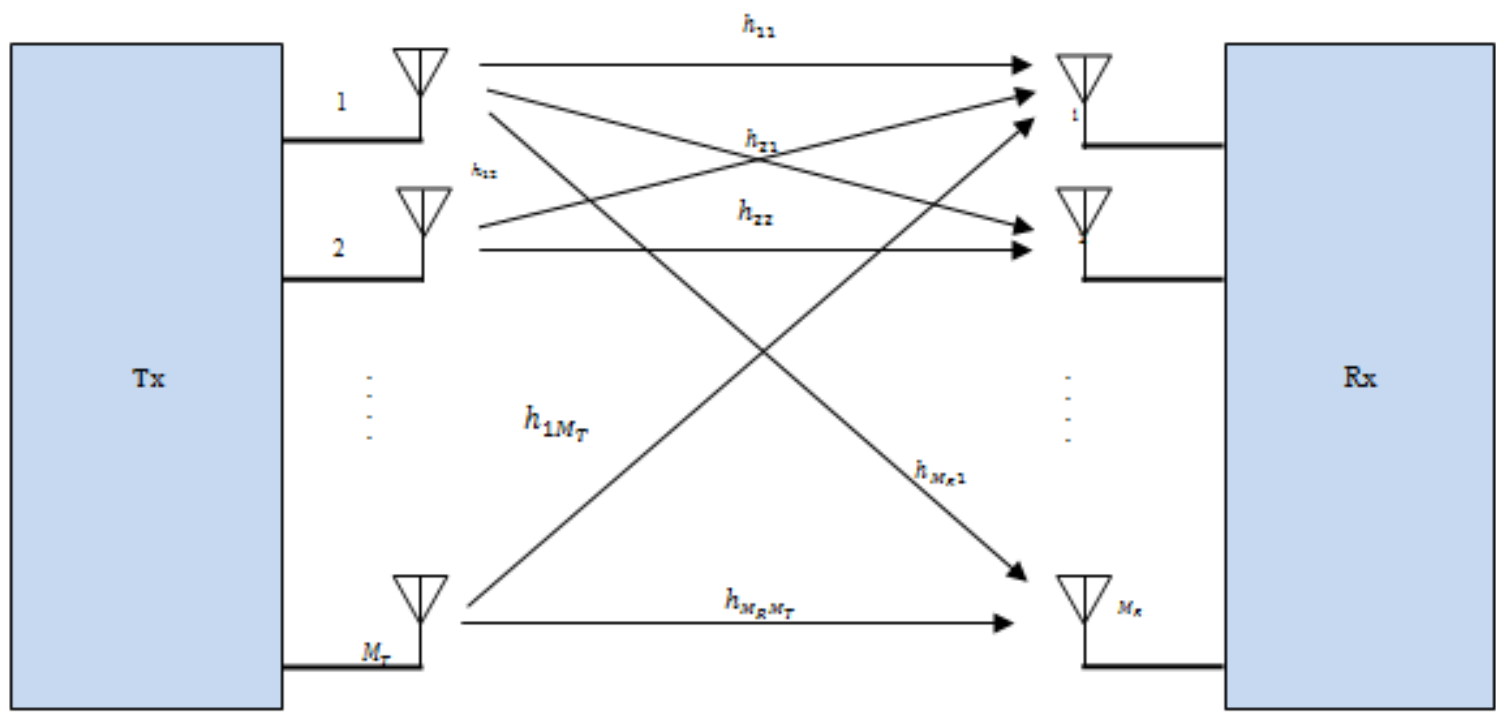

Figure 1: General MIMO System

Volume 5 Issue 1, January 2016 


\section{International Journal of Science and Research (IJSR) \\ ISSN (Online): 2319-7064}

Index Copernicus Value (2013): 6.14 | Impact Factor (2014): 5.611

Multiple antennas can be used at the transmitter and receiver, an arrangement called a MIMO system. A MIMO system takes advantage of the spatial diversity that is obtained by spatially separated antennas in a dense multipath scattering environment. MIMO systems may be implemented in a number of different ways to obtain either a diversity gain to combat signal fading or to obtain a capacity gain.

A MIMO system typically consists of $M_{T}$ transmit and $M_{R}$ receive antennas (Figure 1). By using the same channel, every antenna receives not only the direct components intended for it, but also the indirect components intended for the other antennas. A time-independent, narrowband channel is assumed. The direct connection from antenna 1 to 1 is specified with $h_{11}$, etc., while the indirect connection from antenna 1 to 2 is identified as cross component $h_{21}$, etc. From this is obtained transmission matrix $\mathrm{H}$ with the dimensions $M_{R} \times M_{T}$.

$$
\mathbf{H}=\left[\begin{array}{ccccc}
h_{11} & h_{12} & h & \ldots & h_{1 M_{T}} \\
h_{21} & h_{22} & h & \ldots & h_{2 M_{T}} \\
h_{. .} & h . . & h & \ldots & h_{. M_{T}} \\
h_{M_{R} 1} & h_{M_{R} 2} & h_{n \ldots} & h_{M_{R} M_{T}}
\end{array}\right]
$$

\section{Evolution of MIMO-OFDM}

The history of the MIMO technology dates back to 1984. At that time, Jack Winters of Bell laboratories wrote an article titled "Optimum Combining in Digital Mobile Radio with Co-channel Interference" [1]. After publishing the article a number of efforts have been made by many engineers and academics to better understand the MIMO system.

In 1993 Arogyaswami Paulraj and Thomas Kailath proposed the concept of spatial multiplexing (SM) using MIMO. In the year 1996, few major studies had been made in increasing the signal efficiency over MIMO channels. This year Gregory G. Raleigh and V.K. Jones combinedly wrote a paper titled as "Multivariate Modulation and Coding for Wireless Communication"[2]. In that paper they claimed that multi-path channels can have a multiplicative capacity effect if the multi-path-signal propagation is used in an appropriate communications structure. In the same year, Gerard Joseph Foschini sparked the massive international research effort on multiple-input multiple-output wireless systems that continues till today by introducing the BLAST concept in his paper "Layered Space-Time Architecture for Wireless Communication in a Fading Environment When Using Multi-Element Antennas" [3]. BLAST stands for Bell Laboratories Layered Space-Time. In 1999, Emre Telatar successfully calculated the Shannon capacity of an isotropic fading MIMO channel in his paper, "Capacity of multi antenna Gaussian channels" [4]. He showed that the channel capacity increases with the number of antennas and is proportional to the minimum number of transmit and receive antennas. In 1998, Bell laboratories successfully demonstrated the MIMO system under laboratory conditions. In the following year, Gigabit Wireless Inc. and Stanford University jointly held the first prototype demonstration of MIMO. Eventually in 2002, Lospan Wireless Inc. produced the first commercial product based on MIMO. As of today, several companies have developed
MIMO-OFDM (Orthogonal

frequency-division multiplexing) solution based products. All upcoming 4G systems will be compatible with the MIMO technology[5].

A number of different MIMO systems exist. The optimum decoding of these systems is typically highly complicated. Therefore, a number of decoding algorithms with different complexity-performance tradeoffs have been introduced. Linear detection methods such as Zero-Forcing (ZF) or Minimum Mean Squared Error (MMSE) provide linear complexity, however their performance are suboptimal. Ordered successive interference cancellation decoders such as Vertical Bell Laboratories Layered Space-Time (VBLAST) algorithm, show slightly better performance compared to ZF and MMSE, but suffers from error propagation and is still suboptimal.

MIMO-OFDM combines OFDM with multiple antennas at the transmitter and receiver. This structure permits more prominent diversity when systems are utilized and the process is known as spatial multiplexing which is used for simultaneous data transmission. This process proportionally boosts the information transmission speed by an element equivalent to the amount of transmitting antennas. In addition, since all data is transmitted both in the same frequency band and with separate spatial signatures, this technique utilizes spectrum very efficiently.

MIMO systems can achieve large gains in capacity of communication over wireless channels. For broadband wireless systems, the symbol period becomes smaller relative to the channel delay spread, and therefore we have to cope with frequency-selectivity fading. Large delay spread induces Inter-Symbol Interference (ISI), and cause high performance degradation. So in frequency selective fading channel, OFDM modulation is used to eliminate or reduce the ISI caused by the multipath environments. Combination of MIMO systems with OFDM technology is a promising system for broadband wireless communications. Space-time coded MIMO-OFDM systems have recently attracted much attention for broadband wireless communications including recent IEEE standards $802.11 \mathrm{n}$ and 802.16e. For MIMO-OFDM systems, various spacetime/frequency codes have been developed to achieve both spatial and multipath diversities by coding across subcarriers and multiple antennas and/or across OFDM symbols over the time. However, most of the existing spacetime/frequency codes to achieve the spatial and multipath diversities do not have fast ML decoding. Among the spacetime codes used in MIMO-OFDM systems, OSTBC and Quasi-Orthogonal Space-Time Block Codes (QOSTBC) play important roles since they can achieve full spatial diversity and have fast ML decoding algorithms when the additive noise is white. Recently, a family of space-timefrequency codes have been proposed to achieve the full spatial and multipath diversities for MIMO-OFDM systems and in the meantime they have the fast single-symbol ML decoding by using OSTBC, across multiple antennas and OFDM symbols, and also repeating across subcarriers. Although the repetition across subcarriers can achieve the multipath diversity, it causes high PAPR. This is an important issue for OFDM systems and reducing the PAPR is important in a practical (power efficient) system. One of

\section{Volume 5 Issue 1, January 2016}




\section{International Journal of Science and Research (IJSR) \\ ISSN (Online): 2319-7064 \\ Index Copernicus Value (2013): 6.14 | Impact Factor (2014): 5.611}

the most efficient ways to reduce the PAPR is clipping that, however, induces clipping noise and the induced clipping noise in an MIMO-OFDM system may not be white and thus the fast ML decoding for an OSTBC or QOSTBC coded system may not hold. When the additive noise is not white, ML decoding for spatially colored noised needs to be considered. Many clipping noise mitigation methods have been proposed in the literature. Some of them are based on the decision-aided reconstruction (DAR) and clipping noise cancellation and some of them apply statistical clipping noise models to the ML decoding. All of these methods require the knowledge of the clipping ratio $(\mathrm{CR})$ at the receiver. However, in some applications, for example in interference channels, it is may be possible that the CR is not known at the receiver. A clipping ratio estimation method has been proposed for single antenna OFDM systems in. This clipping ratio estimation method can be applied to pilot-tone-based OFDM systems only.

\section{OFDM for Cognitive Radio}

Interference (ISI). In OFDM, the data is split into several low-rate data chunks and are modulated in overlapping orthogonal subcarriers. These splitting increases the symbol duration by the number of subcarriers used, thus reducing the ISI due to multipath.

OFDM is adapted as the best transmission scheme for Cognitive Radio systems [6]. The features and the ability of the OFDM system makes it fit for the CR based transmission system. OFDM provides spectral efficiency, which is most required for CR system. This is because the subcarriers are very closely spaced and are overlapping, with no interference. Another advantage of OFDM is that it is very flexible and adaptive. The subcarriers can be turned on and off according to the environment and can assist CR system dynamically. OFDM can be easily implemented using the Fast Fourier Transform (FFT), which can be done by digital signal processing using software.

\section{Proposed Methodology}

One of the serious problems of high data rate transmission is time dispersion of pulses resulting in Inter-symbol

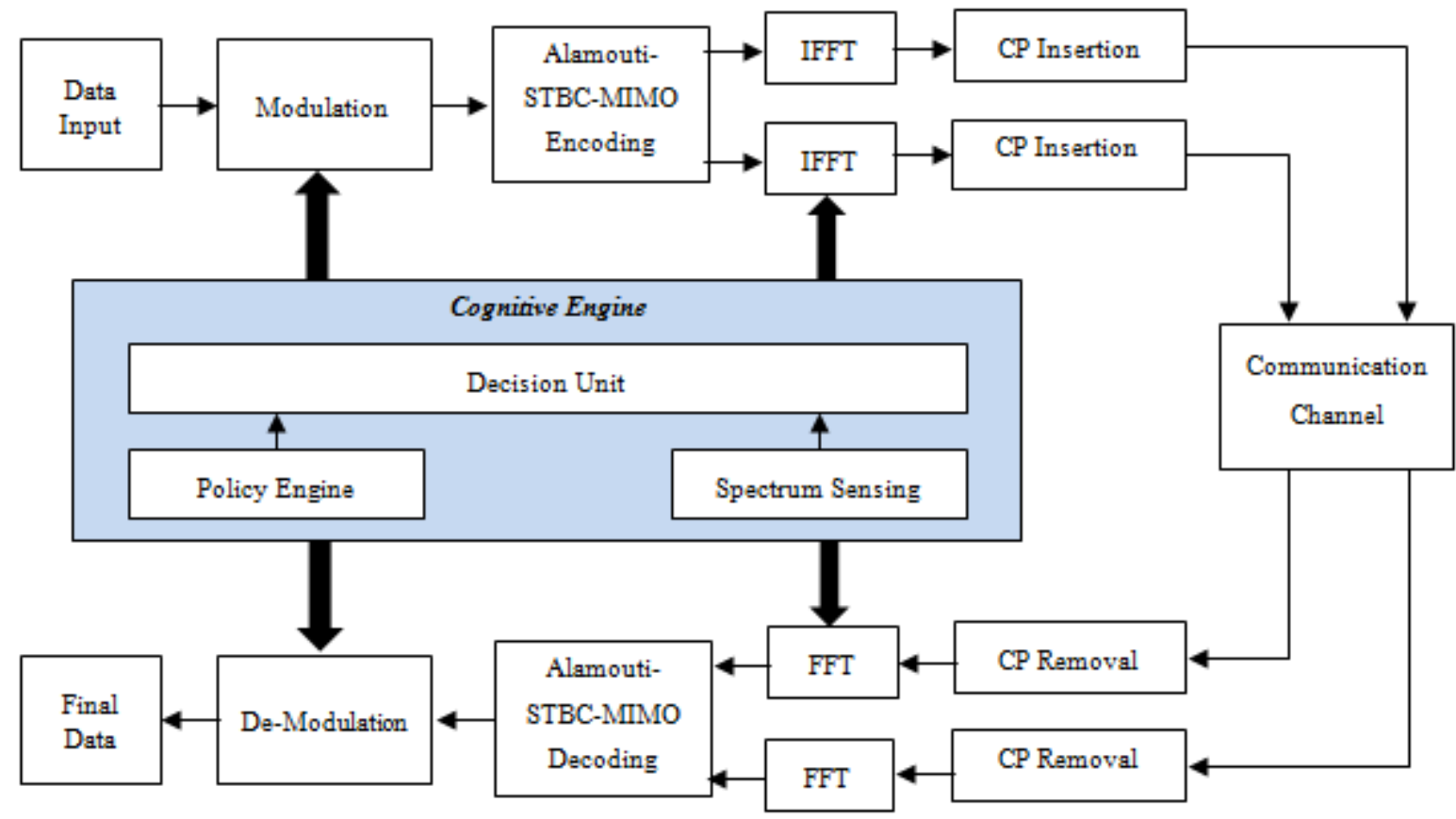

Figure 2: Block diagram for proposed MIMO-OFDM implementation in cognitive scenario

Figure 2 shows the basic block diagram for proposed approach. Modulation is the technique by which the signal wave is transformed in order to send it over the communication channel in order to minimize the effect of noise. This is done in order to ensure that the received data can be demodulated to give back the original data. In proposed system, the high data rate information is divided into small packets of data which are placed orthogonal to each other. This is achieved by modulating the data by a desirable modulation technique (m-array PSK, constellation points; 2, 8, 16 and 64). Then Alamouti-STBC encoding is perform. This scheme has the advantage of achieving a high spatial diversity order in the absence of channel knowledge at the transmitter while keeping the number of receive antennas at the mobile set to a small number. We have concentrated on a two transmitters and two receiver's configuration scheme with the channel model depicted in Figure 3.

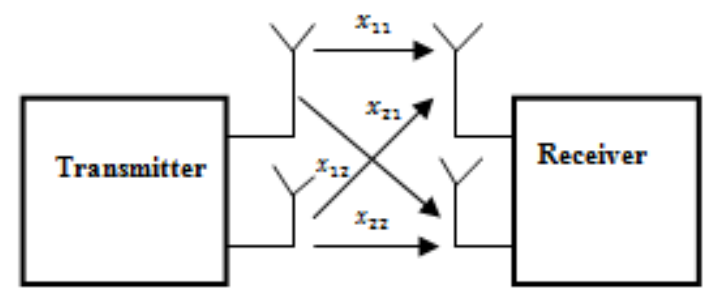

Figure 3: Channel Model for MIMO $(2 \times 2)$

In Figure 3, $x_{11}, x_{12}, x_{21}$ and $x_{22}$ denote the channel's impulses responses between transmit and receive antennas. 


\section{International Journal of Science and Research (IJSR) \\ ISSN (Online): 2319-7064}

Index Copernicus Value (2013): 6.14 | Impact Factor (2014): 5.611

In the Alamouti MIMO scheme [7], diversity is introduced both in space and time to combat the effects of time-varying multipath fading. The diversity is achieved using the Alamouti Space Time Coding where the signal is coded at the transmitter and decoded in the receiver. The symbols, $c_{0}$ and $c_{1}$ are coded at the transmitter according to table 1 .

Table 1: Alamouti Space-Time coding for $2 \times 2 \mathrm{MIMO}$

\begin{tabular}{|l|c|c|}
\hline \multicolumn{3}{c}{ system } \\
\hline time: $\boldsymbol{t}$ & $\boldsymbol{T} \boldsymbol{X}_{\mathbf{1}}$ & $\boldsymbol{T \boldsymbol { X } _ { \mathbf { 2 } }}$ \\
& $c_{0}$ & $c_{1}$ \\
time: $\boldsymbol{t}+\boldsymbol{T}$ & $-c_{1}^{*}$ & $c_{0}^{*}$ \\
\hline
\end{tabular}

Notice that though we are grouping two symbols, we still need two time slots to send two symbols. Hence, there is no change in the data rate. This forms the simple explanation of the transmission scheme with Alamouti Space Time Block coding. After this, IFFT is performed on the encoded signal. In order to avoid ISI, we provide a cyclic prefix to the signal. The Cyclic Prefix or Guard Interval is a periodic extension of the last part of an OFDM symbol that is added to the front of the symbol in the transmitter, and is removed at the receiver before demodulation. Communication channel is the channel through which the data is transferred. Presence of noise in this medium affects the signal and causes distortion in its data content.

Demodulation is the technique by which the original data (or a part of it) is recovered from the modulated signal which is received at the receiver end. In this case, the received data is first made to pass through cyclic prefix removal then FFT of the signal is done. Then STBC decoding is performed and a demodulator is used to get back the original signal. The bit error rate and the signal-to-noise ratio is calculated by taking into consideration the unmodulated signal data and the data at the receiving end (Sink).

\section{Simulation and Results}

The performance of proposed approaches been studied by means of MATLAB simulation.

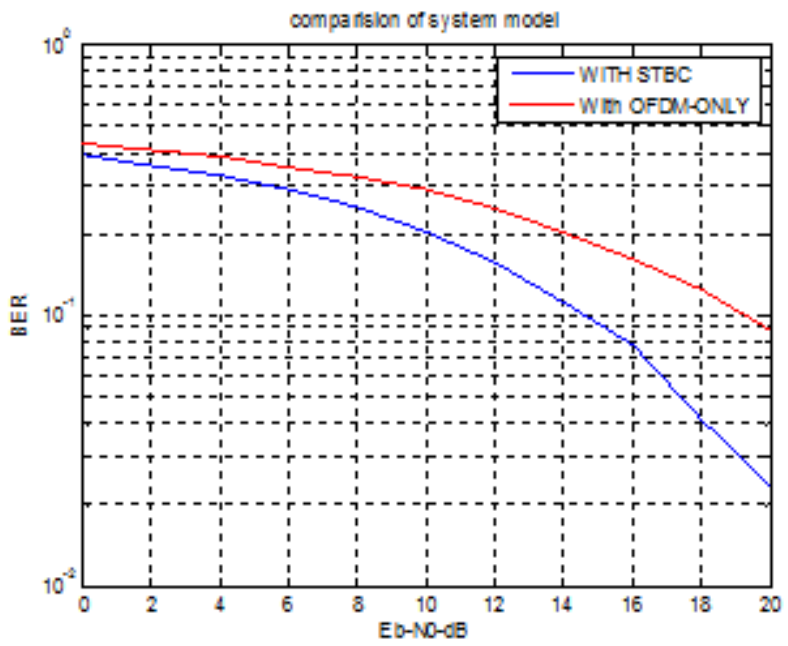

Figure 4: BER performance of proposed system with STBC and OFDM

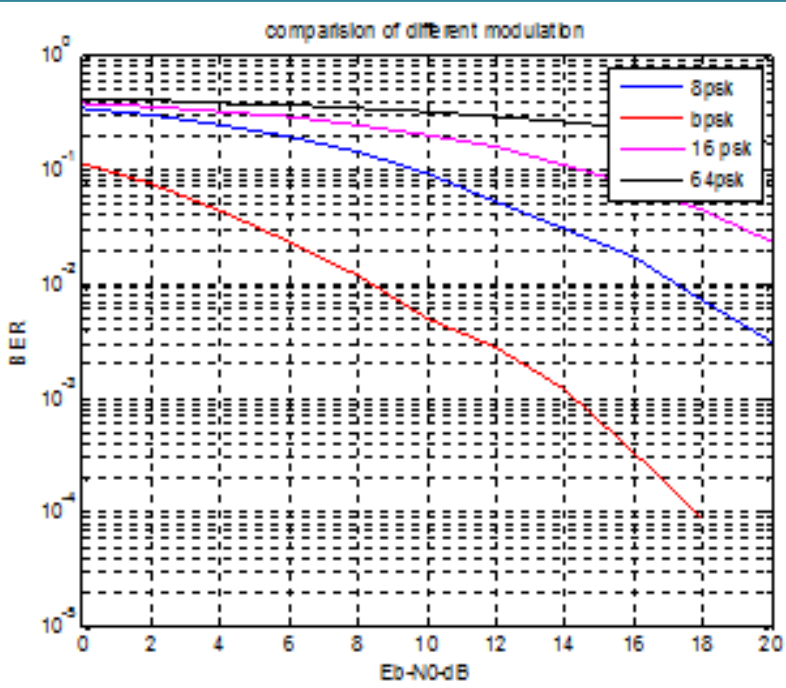

Figure 5: BER performance of proposed system with different modulation techniques

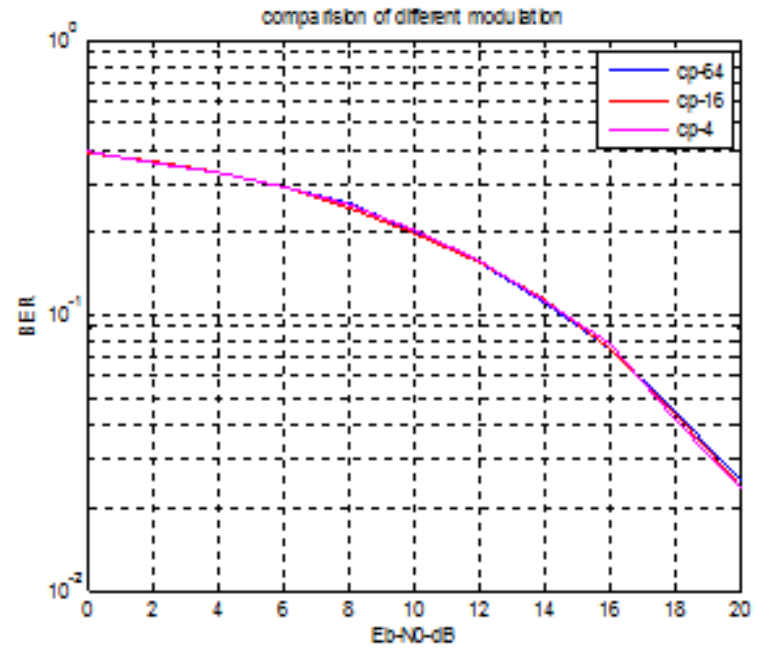

Figure 6: BER performance of proposed system with different cyclic prefix

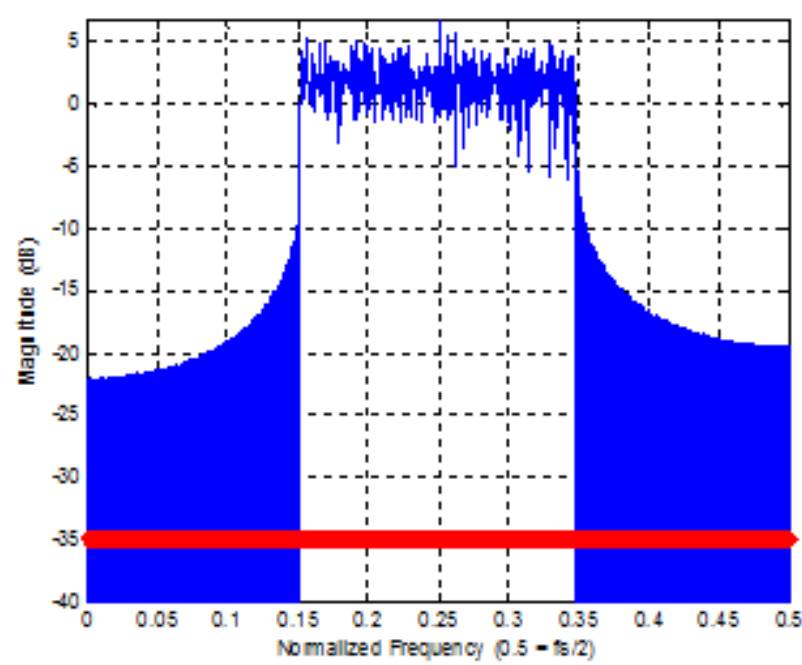

Figure 7: OFDM signal spectrum

\section{Conclusion}

In this paper we have tried to find the BER vs SNR performance with STBC \& OFDM, BER performance for different modulation scheme. Also BER performance is analysis for different values of CP (Cyclic prefix) is also 


\section{International Journal of Science and Research (IJSR) \\ ISSN (Online): 2319-7064}

Index Copernicus Value (2013): 6.14 | Impact Factor (2014): 5.611

studied. In future cognitive radio together with different spectrum sensing method will be simulated and analyzed.

\section{References}

[1] Winters JH.,"Optimum combining in digital mobile radio with co-channel interference", IEEE Journal on Selected Areas in Communications, Vol. 2, Issue 4, July 1984.

[2] Raleigh GG, Jones VK. "Multivariate modulation and coding for wireless communication". IEEE Journal on Selected Areas in Communications, Vol. 17, Issue 5, pp. 851-66, May1999.

[3] Foschini GJ, "Layered space-time architecture for wireless communication in a fading environment when using multi-element antennas", Bell labs technical journal. Vol. 1, Issue 2, pp. 41-59, 1996.

[4] Telatar IE., "Capacity of multi-antenna Gaussian channels", European transactions on telecommunications, Vol. 10, Issue, 6, pp. 585-95, November 1999.

[5] Francisco A. Monteiro, Ian J. Wassell, "Dual-LatticeAided MIMO Detection for Slow Fading Channels", IEEE International Symposium on Signal Processing and Information Technology, Bilbao, Spain, pp. 502507, IEEE Signal Processing Society, Dec 2011.

[6] J. Mitola and G. Q. Maguire, "Cognitive radio: making software radiosmore personal," Personal Communications, IEEE, vol. 6, no. 4, pp. 13-18, Aug. 1999.

[7] S. M. Alamouti, "A simple transmit diversity technique for wireless communications," IEEE J. Sel. Areas Commun., vol. 16, no. 8, pp. 1451-1458, Oct. 1998.

[8] S.Thamizharasan, D. Saraswady, V.Saminadan, "Periodicity based Cyclostationary Spectrum Sensing in Cognitive Radio Networks" International Journal of Computer Applications, Volume 68- No.6, April 2013

[9] Hongjian Sun, Arumugam Nallanathan, Cheng-Xiang Wang, Yunfei Chen "Wideband Spectrum Sensing For Cognitive Radio Networks: A Survey”, IEEE Wireless Communications, 2013.

[10] Jitendra Kumar Saini, Divya Kumar, "Performance Scrutinize of Cyclo-Stationary Detector for OFDM in Cognitive Radio", International Journal of Latest Trends in Engineering and Technology, ISSN: 2278-621X, Issue 3 May 2013. 